UMR 5824

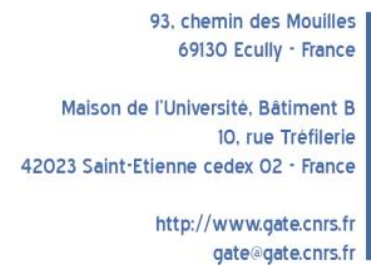

WP 2028 - September 2020

\title{
Stable agreements through liability rules: a multi- choice games approach to the social cost problem
}

\author{
Kevin Techer
}

\begin{abstract}
:
We consider a class of social cost problems where one polluter interacts with an arbitrary number of potential victims. Agents are supposed to cooperate and negotiate an optimal pollution level together with monetary transfers. We examine multi-choice cooperative games associated with a social cost problem and an assignment (or mapping) of rights. We introduce a class of mappings of rights that takes into account the pollution intensity and we consider three properties on mappings of rights: core compatibility, Kaldor-Hicks core compatibility and no veto power for a victim. We demonstrate that there exist only two families of mappings of rights that satisfy core compatibility. However, no mapping of rights satisfies Kaldor-Hicks core compatibility and no veto power for a victim.
\end{abstract}

\section{Keywords:}

Externality, Liability rules, Multi-choice cooperative game, Core

JEL codes:

C71, D62, Q52 


\title{
Stable agreements through liability rules: a multi-choice games approach to the social cost problem
}

\author{
Kevin Techer*†
}

2020

\begin{abstract}
We consider a class of social cost problems where one polluter interacts with an arbitrary number of potential victims. Agents are supposed to cooperate and negotiate an optimal pollution level together with monetary transfers. We examine multi-choice cooperative games associated with a social cost problem and an assignment (or mapping) of rights. We introduce a class of mappings of rights that takes into account the pollution intensity and we consider three properties on mappings of rights: core compatibility, Kaldor-Hicks core compatibility and no veto power for a victim. We demonstrate that there exist only two families of mappings of rights that satisfy core compatibility. However, no mapping of rights satisfies Kaldor-Hicks core compatibility and no veto power for a victim.
\end{abstract}

Keywords: Externality, Liability rules, Multi-choice cooperative game, Core

JEL codes: C71, D62, Q52

\section{Introduction}

A large literature has been developed to describe and solve situations, known as social cost problems, where the activity of some agents has harmful effects on others. This paper aims at precisely analyze the conditions for resolving a social cost problem that involves one polluter and some potential victims. Two traditions stand out to solve a social cost problem: the Pigouvian and the Coasean traditions. The Pigouvian tradition (Pigou $(1920))$ recommends a central intervention by using taxation on the externality. This resulted in the introduction of the polluter-pays principle stating that the polluter should bear the cost of pollution control and prevention measures. The Coasean tradition (Coase (1960)) challenges this polluterpays principle. This tradition argues that agents can solve a social cost problem through a bargaining process provided that property rights are well assigned. The Coase theorem, first formulated by Stigler (1966), summarizes this approach in two theses: first, the efficiency thesis states that in the absence of transaction costs and if property rights are well defined agents will always reach an optimal agreement by bargaining; second, the invariance or

\footnotetext{
${ }^{*}$ Univ Lyon, UJM Saint-Etienne, GATE Lyon Saint-Etienne UMR 5824 , F-42023 Saint-Etienne, France. E-mail: kevin.techer@univ-st-etienne.fr

${ }^{\dagger}$ The author wishes to thank Philippe Solal and Stephane Gonzalez for helpful comments and most relevant references as well as Sylvain Ferrieres for fruitful discussions.
} 
neutrality thesis states that the outcome of the bargaining process is independent of the assignment of rights. Throughout the article, we follow the Coase perspective, and we use cooperative game theory to solve a social cost problem.

The Coase theorem has been analyzed through the scope of cooperative games by Aivazian and Callen (1981) and more broadly by Gonzalez et al. (2019). These articles cover social cost problems which involve more than two agents and formalize the Coase theorem in term of non-emptiness of the core. In the present paper, we extend the framework introduced by Gonzalez et al. (2019) which investigates situations where one polluter interacts with a set of at least two victims. They introduce mappings of rights that constitute the legal structure of negotiation among agents. Specifically, a mapping of rights assigns to each coalition either the value 0 , meaning the coalition is not allowed to negotiate, or 1 if the coalition is allowed to form and negotiate an agreement. This set of mappings of rights can be seen as the set of winning coalition of a proper voting game.1 They propose three properties for mappings of rights: core compatibility which indicates that the core of the cooperative game associated with any social cost problem is nonempty; Kaldor-Hicks core compatibility which indicates that a payoff vector in the core ensures a non-negative payoff to each agent; no veto power for a victim requires that no victim can individually veto an agreement reached by the rest of the society. They show that a mapping of rights satisfies core compatibility if and only if it assigns the rights either to the polluter or to the whole set of victims; while no mapping of rights satisfies those three properties at the same time.

Nonetheless, the set of mappings of rights introduced by Gonzalez et al. (2019) is independent of the polluter's activity level. Either a coalition containing the polluter can negotiate and choose any activity level, or it cannot negotiate. As a result, the model does not take into account the more realistic issue of quotas, which may arise when one wishes to reduce the pollution level. For instance, the US Clean Air Act defines a pollution quota as a limited authorization to emit green house gases but, it does not constitutes a property right on air. Therefore, the right may constitute a restriction on the polluter's activity level as a right of use. In this regard, we introduce the possibility that the right depends on the polluter's activity level. This has three main implications for the model: the conditions defining a mapping of rights must be adjusted; the cooperative game must take into account the different actions of the polluter; and the solution concept that captures the Coase theorem must be adapted in line with the new setting.

To address these points, we modify the conditions defining mappings of rights. Firstly, we impose antitonicity on mappings of rights with respect to the activity level of the polluter. If a coalition containing the polluter is allowed to negotiate an agreement for a certain activity level, then the coalition retains the rights whenever the polluter decreases its activity level. Secondly, we impose monotonicity of a mapping of rights with respect to the participation of victims. If a coalition is allowed to form and negotiate an agreement, it retains the rights when the number of cooperating victims increases. Notice that we can no longer see the set of mapping of rights as generating winning coalitions since a coalition may loose its rights if the activity level increases. This allows us to introduce new mappings of rights which was not taken into account in Gonzalez et al. (2019). Namely, we introduce the mapping of rights that

\footnotetext{
1 Gonzalez et al. (2019) requires three condition for their mappings of rights: the grand coalition is sovereign; if a coalition receives the rights then any larger coalition containing the former inherits the rights (monotonicity); if a coalition receives the rights then nonmembers cannot prevent the coalition to negotiate (effectivity of rights).
} 
assign the rights to the polluter up to any fixed and regulated activity level. This restriction is seen as a pollution quota and the only way for the polluter to override this quota is to reach an agreement with the set of victims. For the sake of simplicity, we suppose the polluter has a finite number of activity levels. Based on these activity levels, each victim can choose whether to participate or not in the negotiation. If the polluter proposes a certain activity level and a subset of victims agrees to participate, then a coalition forms and negotiates an optimal agreement provided they receive the rights to negotiate. These situations where agents have more than one way of acting within a coalition can be modeled using multi-choice games as introduced by Hsiao and Raghavan $(1992,1993)$. While for standard cooperative games each agent has two choices: either to join a coalition and fully participate or not participate at all, in multi-choice games each agent can choose its participation level from a finite set of activity levels. Thus, from each social cost problem endowed with a mapping of rights, we can derive a multi-choice game. In the same vein as Aivazian and Callen (1981) and Gonzalez et al. (2019), we consider the Coase theorem in terms of non-emptiness of the core of this game. Different extensions of the core have been provided for multi-choice games. Here we choose to retain the one introduced and characterized by Grabisch and Xie (2007), which is the set of payoff vector that are efficient regarding each activity level and such that no coalition has incentives to deviate from an agreement reached by the grand coalition. 2 Finally, we naturally extend the properties of core compatibility and Kaldor-Hicks core compatibility. On the other hand we consider no veto power for a victim regarding the highest activity level of the polluter to fit our new framework.

Our main results extend the subset of mappings of rights which guarantee the nonemptiness of the core. We first show that these new mappings of rights that assign quota over the activity of the polluter are core compatible. Each victim perceives the activity of the polluter as a threat which reduces the incentive for victims to free-ride and guarantees the stability of the agreement. Furthermore, any mapping of rights that exclusively assigns the rights to subsets of victims and the grand coalition is core compatible. Since not all mapping of rights are core compatible, this result invalidates the neutrality thesis of the Coase theorem. We show that the unique way to satisfy core compatibility and no veto power for a victim is to assign the rights to the polluter at its highest activity level. On the contrary, the only mappings that satisfy Kaldor-Hicks core compatibility assign the rights to a subset of victims. Finally, we confirm the impossibility result regarding Kaldor-Hicks core compatibility and no veto power for a victim at the same time.

\section{Related literature:}

The Coase theorem has encouraged the emergence of a large literature, in particular because Coase considers only two agents and does not provide any formal model. That literature has mainly focused on three types of models. The first type considers the theorem in terms of competitive equilibrium. For instance Hurwicz (1995) and Chipman and Tian (2012) provide necessary and sufficient conditions for the validity of the Coase theorem for situations involving two agents. Both papers highlights the pivotal role of parallel preferences that ensure the validity of the neutrality thesis. The second type incorporates strategic interactions between agents and formulates the Coase theorem in terms of Nash equilibrium of a strate-

\footnotetext{
${ }^{2}$ For other extensions of the core, one can refer to van den Nouweland et al. (1995) and Hwang and Liao (2011).
} 
gic/bargaining game: Anderlini and Felli (2001) analyzes negotiations between two agents in the presence of transaction costs; Ellingsen and Paltseva (2016) highlights the possibility of free-riding behaviors when more than two agents are involved. This paper aligns with the third type of models which consider cooperative game theory. A famous instance is the Shapley and Shubik (1969) garbage game where a finite set of neighbors have to decide on where to locate their garbage. Using a simple game authors show that the core of the garbage game is non-empty if and only if no more than two agents are involved. In the same spirit, Ambec and Kervinio (2016) analyzes the Not In My Back Yard (NIMBY) problem. In comparison to the garbage game, the NIMBY problem consider the location of a locally undesirable but globally desirable facility. Authors propose a spatial model with externalities, and analyze under which conditions the Coase theorem is likely to hold. They also provide an index for testing the non-emptiness of the core of a game associated with a NIMBY problem. Zhao (2018) proposes a solution for the empty-core problem introduced by Aivazian and Callen (1981). The author argues that the set of agents does not necessarily split the worth of the grand coalition but can organize themselves into the most beneficial collection of coalitions.

The rest of the paper is organized as follows. Section 2 presents the formal framework within which we define the social cost problem and the mappings of rights along with the corresponding multi-choice game. Section 3 presents our main result, and Section 4 provides an illustration with simple spatial considerations.

\section{Preleminaries}

\subsection{Multi-choice cooperative games and the core}

Let $N:=\{1, \ldots, n\}$ be a finite set of agents. Each agent $i \in N$ is endowed with a finite number of participation levels. The worth the set of agents can obtain depends on the participation level of the cooperating agents. Let $L_{i}:=\left\{0,1, \ldots, l_{i}\right\}$ be the set of participation levels agent $i \in N$ is able to undertake, where 0 means that agent $i$ does not participate. For each $S \subseteq N, S \neq \emptyset$, let $L_{S}$ be the product set $L_{S}=\times_{i \in S} L_{i}$; we will write $L$ instead of $L_{N}$. Elements $x$ of $L$ are called multi-choice coalitions or participation profiles, we will use both terms without distinction. The notation $\left(x_{-i}, k_{i}\right) \in L_{-i} \times L_{i}$ stands for the participation profile where each agent $j \neq i$ chooses the level $x_{j} \in L_{j}$ besides agent $i$ which chooses $k_{i} \in L_{i}$. For any $S \subseteq N$, let $-S:=N \backslash S$, we will sometimes use the notation $\left(x_{-S}, x_{S}\right) \in L_{-S} \times L_{S}$ in order to insist on the participation levels chosen by the sets of agents $S$ and $-S$. For any two participation profiles $x$ and $y$ we write: $x \leq y$ if $x_{1} \leq y_{1}, \ldots, x_{n} \leq y_{n}$ and we have $x \vee y=$ $\left(x_{1} \vee y_{1}, \ldots, x_{n} \vee y_{n}\right)=\left(\max \left\{x_{i}, y_{i}\right\}\right)_{i \in N}$, and $x \wedge y=\left(x_{1} \wedge y_{1}, \ldots, x_{n} \wedge y_{n}\right)=\left(\min \left\{x_{i}, y_{i}\right\}\right)_{i \in N}$, where $x \vee y$ and $x \wedge y$ denote the supremum and infimum respectively.

For $x \in L$ we define the support of $x$ by

$$
\mathcal{S}(x):=\left\{i \in N \mid x_{i}>0\right\},
$$

$\mathcal{S}(x)$ is the group of active agents in the participation profile $x \in L$. The characteristic function $v: L \rightarrow \mathbb{R}$ is any function such that $v(0, \ldots, 0)=0$, which assigns to each participation profile $x \in L$ the worth that agents can obtain when each agent $i \in N$ chooses to participate at level $x_{i} \in L_{i}$.

A multi-choice game is a triple $(N, L, v)$, where $N$ is the set of agents, $L$ is the set of participation profiles, and $v$ is the characteristic function. Throughout the article $N$ and $L$ are 
fixed, therefore we will denote a multi-choice game by $v$. We denote by $M C$ the class of multi-choice games.

Let $v \in M C$. We define $M:=\left\{(i, k) \mid i \in N, k \in L_{i}\right\}$. A payoff vector $\psi$ is an element of $\mathbb{R}^{M}$, whose coordinates are denoted by $\psi_{(i, k)}$. Thus, a payoff vector describes the payoff that each agent receive for each participation level. By convention for each agent $i \in N, \psi_{(i, 0)}=0$. For any $x \in L$, define the total payoff $\psi(x)$ as:

$$
\psi(x)=\sum_{i \in N} \psi_{\left(i, x_{i}\right)} .
$$

The core is the most popular set-valued solution concept in cooperative game theory. It corresponds to the set of efficient payoffs such that no group of agents has an incentive to split off from the grand coalition and to form a smaller group, because they collectively receive a payoff greater than or equal to what they can obtain by agreeing to cooperate in this smaller group. Here, we consider the core of a multi-choice game as defined by Grabisch and Xie (2007) ${ }^{3}$

Definition 1. The core of a multi-choice game $v \in M C$ on $L$, denoted by $\mathcal{C}(v)$, is defined as

$$
\begin{aligned}
\mathcal{C}(v):=\{ & \left\{\psi \in \mathbb{R}^{M} \mid \forall x \in L, \psi(x) \geq v(x)\right. \text { and } \\
& \left.\forall k=1, \ldots, \max _{j} l_{j}, \psi\left(k \wedge l_{1}, \ldots, k \wedge l_{n}\right)=v\left(k \wedge l_{1}, \ldots, k \wedge l_{n}\right)\right\} .
\end{aligned}
$$

Each payoff vector in the core drives agents to align on the participation level $\left(k \wedge l_{1}, \ldots, k \wedge\right.$ $\left.l_{n}\right) \in L$, where $k=1, \ldots, \max _{j} l_{j}$, as far as possible for them. For instance, one can think of a set of agents which have to cooperate in some project. It would be desirable that all agents make efforts and participate in the project. It could also be desirable that no agent participate less than others within the project. However, it can be the case that agents are not able to provide the same level of participation, let say because of some legal constraints. Choosing a payoff vector in the core, whenever it is non-empty, ensures a consensus for each participation level. If agents agree on the participation level $k=4$ while for some agent $j \in N$ the highest participation level is $l_{j}=2$, then no agent $i \in N$ has incentives to make an effort different from $k_{i}=4$ if it is able to, otherwise it chooses the maximum effort available $\left(l_{j}=2\right)$.

Grabisch and Xie (2007) extend the Bondareva-Shapley theorem (Bondareva (1962), Shapley (1967)) for the multi-choice setting, that provides a necessary and sufficient condition for the non-emptiness of the core of a multi-choice game in the following way.

Definition 2. A collection $\mathcal{B}$ of elements of $L \backslash\{(0, \ldots, 0)\}$ is balanced if there exist positive coefficients $\mu(x), x \in L$ such that

$$
\begin{aligned}
\forall i \in N, \forall k=1, \ldots, l_{i}-1, \sum_{\substack{x \in B \\
x_{i}=k}} \mu(x)=1, \\
\forall i \in N, \sum_{\substack{x \in B \\
x_{i}=l_{i}}} \mu(x)=\left(\max _{j \in N} l_{j}\right)-l_{i}+1 .
\end{aligned}
$$

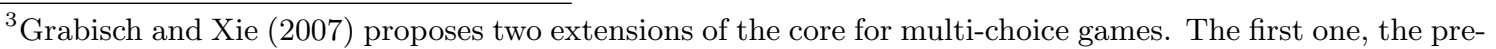
core is a natural extension of the notion of the core. However, the pre-core may be an unbounded polyhedron. We prefer to analyze the second definition of the core where each individual payoff is bounded. 
A natural extension of the standard interpretation of balanced collection can be given as follows. Consider there $\operatorname{are~}_{\max _{j}} l_{j}$ working days, where each day is dedicated to one activity level $k=1, \ldots, \max _{j} l_{j}$. For each day $k<l_{i}$, (1) holds: agent $i$ in $N$ allocates one unit of time among configurations $x \in L \backslash\{(0, \ldots, 0)\}$ such that $x_{i}=k$. From day $k=l_{i},(2)$ holds and it remains $\left(\max _{j \in N} l_{j}\right)-l_{i}+1$ days. Then $i$ allocates the rest of its time among configurations $x \in L \backslash\{(0, \ldots, 0)\}$ such that $x_{i}=l_{i}$. A collection of element of $L \backslash\{(0, \ldots, 0)\}$ is balanced if each agent $i \in N$ fully allocates these $\max _{j} l_{j}$ units of time as described.

Definition 3. A multi-choice game $v \in M C$ is balanced if for every balanced collection $\mathcal{B}$ of elements of $L \backslash\{0, \ldots, 0\}$ with coefficients $\mu(x), x \in \mathcal{B}$, it holds

$$
\sum_{x \in \mathcal{B}} \mu(x) v(x) \leq \sum_{k=1}^{\max _{j} l_{j}} v\left(k \wedge l_{1}, \ldots, k \wedge l_{n}\right) .
$$

Proposition 2.1 (Grabisch and Xie (2007)). A multi-choice game $v \in M C$ has a non-empty core if and only if it is balanced.

\subsection{Social cost problems}

Let $p$ be a polluter and denotes by $U$ a finite set of at least two potential victims. The set $N=U \cup\{p\}$ denotes the finite set of agents involved in a social cost problem, $N$ is supposed to fixed. The polluter chooses a participation level it wishes to operate in the fixed set $L_{p}:=\left\{0,1,2, \ldots, l_{p}\right\}$ which corresponds to its set of activity levels. Each victim $i \in U$ decides whether or not to take part in the cooperation; hence the action set for each victim is $L_{i}:=\{0,1\}$. Let $L:=\times_{i \in N} L_{i}$, an element $x:=\left(x_{1}, \ldots, x_{n}, x_{p}\right) \in L$ is a multi-choice coalition/participation profile. The activity of the polluter generates a private benefit for itself represented by a non-negative and non-decreasing function $B_{p}: L_{p} \rightarrow \mathbb{R}_{+}$. However, this activity causes damage for each victim $i \in U$, represented by a non-decreasing and nonnegative function $D_{i}: L_{p} \rightarrow \mathbb{R}_{+}$. For each $i \in N$, we assume that $B_{p}(0)=D_{i}(0)=0$. We summarize a social cost problem by the data $P:=\left(N, L, B_{p},\left(D_{i}\right)_{i \in U}\right)$. Let $\mathcal{P}$ be the class of all social cost problems $P$.

Given a social cost problem $P \in \mathcal{P}$, for each participation profile $x \in L$, we denote by $K_{x}^{*}$ the non-empty set of optimal activity levels

$$
K_{x}^{*}:=\arg \max _{k \in\left[\left[0, x_{p}\right]\right]}\left\{1_{p}(x) B_{p}(k)-\sum_{\substack{i \in N \\ x_{i}=1}} D_{i}(k)\right\},
$$

where $\left[\left[0, x_{p}\right]\right]$ denotes the set $\left\{0,1, \ldots, x_{p}\right\} \subseteq L_{p}, 1_{p}(x)=1$ if $x_{p}>0$ and $1_{p}(x)=0$ otherwise. We denote by $k_{x}^{*}$ an element of $K_{x}^{*}$. Note that $0 \in K_{x}^{*}$ whenever $x \in L$ is such that $x_{p}=0$.

\subsection{Mapping of rights}

Given a participation profile $x \in L$, we consider a right as an authorization for the group of active agents $\mathcal{S}(x)$ to form and negotiate a binding agreement when each active agent chooses its participation level $x_{i} \in L_{i}$. In this way, rights are related to the participation profiles rather than to groups of agents exclusively. 
A mapping of rights $\phi: L \rightarrow\{0,1\}$ assigns to each participation profile $x \in L$ the value 0 or 1 . The value $\phi(x)=0$ means that the group of active agents in this participation profile does not have the rights to negotiate an agreement. The value $\phi(x)=1$ means that the group of active agents is allowed to form a coalition and negotiate. We impose the following conditions on mappings of rights:

$$
\phi(0, \ldots, 0)=0 \text { and } \phi\left(1_{-p}, l_{p}\right)=1
$$

(C2) for each $x_{p} \in L_{p}$, it holds that:

$$
\left[\forall x_{-p}, x_{-p}^{\prime} \in L_{-p}, x_{-p} \leq x_{-p}^{\prime}\right] \Rightarrow\left[\phi\left(x_{-p}, x_{p}\right) \leq \phi\left(x_{-p}^{\prime}, x_{p}\right)\right],
$$

(C3) for each $x_{-p} \in L_{-p}$, it holds that:

$$
\left[\forall x_{p}, x_{p}^{\prime} \in L_{p} \backslash\{0\}, x_{p} \leq x_{p}^{\prime}\right] \Rightarrow\left[\phi\left(x_{-p}, x_{p}\right) \geq \phi\left(x_{-p}, x_{p}^{\prime}\right)\right],
$$

(C4) for each $S \subseteq N$, and each $\left(0_{-S}, x_{S}\right) \in L_{-S} \times L_{S}$, it holds that:

$$
\left[\phi\left(0_{-S}, x_{S}\right)=1\right] \Rightarrow\left[\forall x_{-S} \in L_{-S}, \phi\left(x_{-S}, 0_{S}\right)=0\right] .
$$

(C1) states that if agents are unwilling to make any participation they do not receive any rights. By contrast, if they all endeavor full participation, then they receive the rights. Therefore, full participation implies sovereignty.

(C2) translates the idea of monotonicity of $\phi$ on $L_{-p}$ for any fixed participation level of the polluter $x_{p} \in L_{p}$. Any group of active agents that receives the rights should retain these rights when more victims become active. Notice that, in the model of Gonzalez et al. (2019), the mapping of rights is monotonic with respect to set inclusion. In contrast, we propose that a group of active agents should preserves its rights only when new victims become active. However, it might be possible that a group of active agents loose its rights if the polluter becomes active or if it increases its activity level.

(C3) relates to participation profiles where the polluter is active and translates the idea of antitonocity of $\phi$ on $L_{p} \backslash\{0\}$. If a group of active agents is not allowed to form a coalition and negotiate when $x_{p} \in L_{p} \backslash\{0\}$, i.e $\phi\left(x_{-p}, x_{p}\right)=0$, the group still not receives the rights when the polluter increases its activity level, that is when $x_{p} \leq x_{p}^{\prime}$ then $\phi\left(x_{-p}, x_{p}^{\prime}\right)=0$. On the contrary, it may be the case that this group receives the rights when the polluter decreases its activity level. In the case where $\phi\left(x_{-p}, x_{p}\right)=1$, it retains the rights for a lower activity level, that is when $x_{p}^{\prime \prime} \leq x_{p}$ then $\phi\left(x_{-p}, x_{p}^{\prime \prime}\right)=1$.

Finally, (C4) describes the effectivity of the rights. Consider a participation profile $x \in$ $L \backslash\{(0, \ldots, 0)\}$ such that $\mathcal{S}(x)=S \subseteq N$ and $\phi(x)=1$. Whatever the participation level $x_{-S} \in L_{-S}$ of agents in $-S$, if all agents in $S$ become inactive, then the corresponding participation profile does not receive the rights: $\phi\left(x_{-S}, 0_{S}\right)=0$. Thus, it cannot hold that $S$ and $-S$ receives the right at the same time.

By combining (C1) with (C3) we obtain that the whole society $N$ has the right to negotiate whatever the activity level $k_{p} \in L_{p} \backslash\{0\}$ chosen by the polluter, $\phi\left(1_{-p}, k_{p}\right)=1$. In other words, the whole society is always sovereign.

We denote by $\Phi$ the class of mapping of rights that satisfy the above conditions ${ }^{4}$

\footnotetext{
${ }^{4}$ One can verify that the class of mapping of rights introduced in Gonzalez et al. (2019) can be defined using our new conditions, therefore our class of mapping of rights constitutes a generalization.
} 
Example 1. Consider the mapping which gives the rights to each majority coalition. In the multi-choice game framework, this mapping of rights becomes $\phi^{m a j} \in \Phi$, defined as follows:

$$
\phi^{m a j}(x)=\left\{\begin{array}{cc}
1 & \text { if }|\mathcal{S}(x)|>n / 2 \\
0 & \text { otherwise }
\end{array}\right.
$$

One can easily verify that $\phi^{m a j} \in \Phi$ satisfies each condition defining a mapping of rights.

\subsection{Multi-choice games and mapping of rights}

This section deals with the derivation of a multi-choice game with respect to a social cost problem $P=\left(N, L, B_{p},\left(D_{i}\right)_{i \in U}\right) \in \mathcal{P}$ and a mapping of right $\phi \in \Phi$. Conflicting interests and externalities in a social cost problem are such that the worth a group of agents can obtain depends on the behavior of other agents. Due to external effects, when a group of agents decides to be active, each member of the group may form diverse expectations regarding the behavior of the remaining agents. Such expectations relate to how the remaining agents will organize themselves into groups $5^{5}$ Hence, the construction of the multi-choice cooperative game is closely related to the formation of expectations.

We assume that agents form pessimistic expectations regarding the behavior of the remaining agents. Let $x \in L$ be a participation profile. By "pessimistic" we mean that, when a group of active agents $\mathcal{S}(x)=S \subseteq N$ chooses the participation profile $x_{S} \in L_{S}$, active agents $i \in S$ expect that the remaining agents, in $-S$, will organize themselves in order to create the worst scenario for $S$ (with respect to the mapping of right $\phi \in \Phi$ ). Notice that this assumption is not without significance since it is the most favorable one for the non-emptiness of the core6

Formally, for any participation profile $x=\left(0_{-S}, x_{S}\right) \in L$, where $S=\mathcal{S}(x)$, we associate the set of complementary participation profiles defined as

$$
\bar{C}_{x}:=\left\{\left(y_{-\mathcal{S}(x)}, 0_{\mathcal{S}(x)}\right) \in L \mid y_{-\mathcal{S}(x)} \in \times_{i \in-\mathcal{S}(x)} L_{i}\right\}
$$

Each element of $\bar{C}_{x}$ corresponds to a participation profile where each agent $i \in S$ is inactive. The worst response for $x \in L$, denoted by $w_{x} \in \bar{C}_{x} \subseteq L$, is a participation profile such that:

$$
k_{w_{x}}^{*}= \begin{cases}\max _{y \in \bar{C}_{x}}\left\{k_{y}^{*}\right\} & \text { if } \exists y \in \bar{C}_{x} \text { such that } \phi(y)=1, \\ 0 & \text { otherwise }\end{cases}
$$

where $k_{y}^{*} \in K_{y}^{*}$ is the optimal activity level for $y \in \bar{C}_{x}$. If $\phi(x)=1$, by (C4) each agent invovled in the participation profile $x \in L$ knows that the remaining agents cannot prevent them to negotiate. Then there is no credible worst response.

\footnotetext{
${ }^{5}$ See Bloch and Van den Nouweland $(2014)$ for an axiomatic analysis of expectation formation rules.

6 Funaki and Yamato (1999) analyze the tragedy of commons through cooperative games in partition form. Authors prove that the core of a common pool resource game is always non-empty under the assumption of pessimistic expectations.
} 
Given a social cost problem $P \in \mathcal{P}$ and a mapping of rights $\phi \in \Phi$, the associated multi-choice game $v_{\phi, P} \in M C$ is defined as follows,

$$
v_{\phi, P}(x)=\left\{\begin{array}{cc}
\max _{k \in\left[\left[0, x_{p}\right]\right]}\left(B_{p}(k)-\sum_{i \in \mathcal{S}(x) \backslash\{p\}} D_{i}(k)\right) & \text { if } \phi(x)=1, \text { and } \mathcal{S}(x) \ni p, \\
-\sum_{i \in \mathcal{S}(x)} D_{i}\left(k_{w_{x}}^{*}\right) & \text { if } \phi(x)=0, \text { and } \mathcal{S}(x) \not \supset p, \\
0 & \text { otherwise. }
\end{array}\right.
$$

Let $x \in L$ be a participation profile such that $\phi(x)=1$ and $\mathcal{S}(x) \ni p$. Active agents can select any socially optimal activity level in $K_{x}^{*}$. By $(\mathrm{C} 4)$ the rights are effective and active members, $\mathcal{S}(x)$, do not have to worry about the behavior of the remaining agents therefore

$$
v_{\phi, P}(x)=\max _{k \in\left[\left[0, x_{p}\right]\right]}\left(B_{p}(k)-\sum_{i \in \mathcal{S}(x) \backslash\{p\}} D_{i}(k)\right) .
$$

If $x \in L$ is such that $\phi(x)=0$ and $\mathcal{S}(x) \not \supset p$. In other words, only victims decide to be involved in the cooperation, $\mathcal{S}(x) \subseteq U$, but they do not receive the rights. By the pessimistic expectations assumption, active agents expect the worst response by the remaining agents including the polluter. Therefore each agent in $\mathcal{S}(x) \subseteq U$ expects the activity level $k_{w_{x}}^{*}$.

The last case contains two parts. Either $x \in L$ is a participation profile such that $\phi(x)=0$ and $\mathcal{S}(x) \ni p$. Then, the worst scenario active agents expect from the outsiders is $k_{w_{x}}^{*}=0$. Hence the worth of the participation profile is $v_{\phi, P}(x)=0$. Or, $x \in L$ is such that $\phi(x)=1$ where only active victims are involved $\mathcal{S}(x) \not \supset p$. Then, by the effectivity of rights $(\mathrm{C} 4)$ the remaining agents cannot prevent $\mathcal{S}(x)$ to exercise the rights so $v_{\phi, P}(x)=0$.

\subsection{Properties for mappings of rights}

Given a mapping of rights $\phi \in \Phi$ and a social cost problem $P \in \mathcal{P}$, the outcome of the negotiation among agents is represented by a payoff vector $\psi \in \mathbb{R}^{M}$ of the multi-choice game $v_{\phi, P}$. In this section, we present some desirable properties for mappings of rights. The first one is related to the existence of core elements for the multi-choice game derived from a mapping of rights $\phi \in \Phi$ and a social cost problem $P \in \mathcal{P}$. The second properties relates the feasibility of payoff vectors in the core and the full compensation principle. This property strengthen the later imposing a positive payoff for each agent whatever the participation level. Finally the last property considers the rights themselves.

Definition 4. A mapping of rights $\phi \in \Phi$ satisfies core compatibility if for each social cost problem $P \in \mathbb{P}, \mathcal{C}\left(v_{\phi, P}\right) \neq \emptyset$.

This first property is straightforward. The game associated with a mapping of rights that satisfies core compatibility always has a non-empty core regardless the social cost problem $P \in \mathcal{P}$. Therefore whatever the benefit function $B_{p}$ or the damage functions $D_{i}$ for each victim $i \in U$, the core of the social cost game is non-empty.

Definition 5. A mapping of rights $\phi \in \Phi$ satisfies Kaldor-Hicks core compatibility if for each social cost problem $P \in \mathbb{P}, \mathcal{C}\left(v_{\phi, P}\right) \cap \mathbb{R}_{+}^{M} \neq \emptyset$. 
The Kaldor-Hicks core compatibility property imposes a non-negative payoff for each agent at each participation level. In our context, the polluter is the only agent who can have different activity levels. Hence Kaldor-Hicks core compatibility imposes that each victim should receive a non-negative payoff for its participation in order to be fully compensated for the damage. Also, the polluter should receive a non-negative payoff for each of its activity level.

Definition 6. A mapping of rights satisfies no veto power for a victim if for each victim $i \in U, \phi\left(1_{-i, p}, 0_{i}, l_{p}\right)=1$.

According to this property, if a victim decides to leave the grand coalition by becoming inactive, the participation profile should not be affected regarding the law. Notice that, by (C3) if a mapping of rights $\phi \in \Phi$ satisfies no veto power for a victim, each participation profile $\left(1_{-i, p}, 0_{i}, x_{p}\right) \in L$ such that $\mathcal{S}\left(1_{-i, p}, 0_{i}, x_{p}\right)=N \backslash\{i\}$ retains the rights: $\phi\left(1_{-i, p}, 0_{i}, x_{p}\right)=1$. By (C4), each victim $i \in U$ alone do not have the power to prevent the negotiation among the remaining agents $\phi\left(0_{-i}, 1_{i}\right)=0$. Since we can consider a large set of victims, this property can be seen as a weak democratic principle across the set of victims. Indeed, one isolate victim cannot impose her choice to the remaining agents. No victim should have more power than other victims regarding the legal framework.

Example 2. Consider the following social cost problem $P \in \mathcal{P}$ defined as follows. Let $N:=\{1,2, p\}$ be the set of agents, $L_{p}:=\{0,1,2,3,4\}$ is the action set of the polluter, $L_{1}=L_{2}=\{0,1\}$, and

$$
B_{p}\left(x_{p}\right)=4 \sqrt{x_{p}}, \quad D_{1}\left(x_{p}\right)=\frac{6}{10} \times 2 x_{p}, \quad D_{2}\left(x_{p}\right)=\frac{3}{10} \times 2 x_{p} .
$$

Consider the mapping of rights $\phi^{m a j} \in \Phi$ as introduced in Example 1. The associated multichoice game $v_{\phi^{m a j}, P} \in M C$ is as follows:

$$
v_{\phi^{m a j}, P}(x)=\left\{\begin{array}{cl}
4 \sqrt{k_{x}^{*}}-\sum_{\substack{i \in U \\
x_{i}=1}} D_{i}\left(k_{x}^{*}\right) & \text { if } \mathcal{S}(x) \ni p,|\mathcal{S}(x)| \geq 2, \\
0 & \text { otherwise. }
\end{array}\right.
$$

We can show that $\mathcal{C}\left(v_{\phi^{m a j}, P}\right)=\emptyset$ by considering the balanced collection $\mathcal{B}$ defined as

$$
\mathcal{B}:=\{x \in L:|\mathcal{S}(x)|=2\},
$$

with the balancing weights:

$$
\mu(x)=\frac{4}{2}, \text { if } x=(1,1,0), \text { and } \mu(x)=\frac{1}{2} \text { otherwise. }
$$

It should be noticed that any agreement reached by the grand coalition necessarily bring a lower worth than the one reached by any two agents coalition. By simple computations we have that:

$$
\sum_{x \in \mathcal{B}} \mu(x) v_{\phi^{m a j}, P}(x)=15.64>8.8=\sum_{k=1}^{4} v_{\phi^{m a j}, P}(1,1, k) .
$$

By proposition 2.1, we conclude that the core of the game is empty. 


\section{Core compatible mappings of rights}

\subsection{Restriction on the polluter activity level}

In order to reduce the pollution level, it might be preferable to control the activity of the polluter. For instance, one can imagine that agents have a primal agreement on the pollution level that constraints the polluter up to a given threshold. In this perspective, we consider mappings of rights that allow the polluter to produce up to a fixed "regulated activity level" $\tilde{k}_{p} \in L_{p} \backslash\{0\}$. The idea behind this family of mappings of rights is to reconsider the responsibility of the polluter. Up to the regulated level $\tilde{k}_{p} \in L_{p}$, the polluter should not be responsible for the damage its activity creates. Beyond $\tilde{k}_{p}$, the mapping of rights should allowed negotiations provided that all victims are involved, meaning that unanimity is required to sign an agreement. In other words, only the grand coalition is able to negotiate for an activity level greater than $\tilde{k}_{p}$. We formally define this type of mapping of rights $\phi_{\tilde{k}_{p}}$ as follows:

$$
\phi_{\tilde{k}_{p}}(x)= \begin{cases}1 & \text { if } \mathcal{S}(x) \ni p \text { and } 0<x_{p} \leq \tilde{k}_{p}, \text { or } \mathcal{S}(x)=N \\ 0 & \text { otherwise. }\end{cases}
$$

We denote by $\tilde{\Phi} \subseteq \Phi$ this family of mapping of rights.

Given a social cost problem $P \in \mathcal{P}$, a mapping of rights $\phi_{\tilde{k}_{p}} \in \tilde{\Phi}$, we derive the corresponding multi-choice game $v_{\hat{k}_{p}, P} \in M C$ from (4) as follows:

$$
v_{\phi_{\tilde{k}_{p}}, P}(x)=\left\{\begin{array}{cl}
B_{p}\left(k_{x}^{*}\right)-\sum_{\substack{i \in U \\
x_{i}=1}} D_{i}\left(k_{x}^{*}\right) & \text { if } \mathcal{S}(x) \ni p \text { and } 0<x_{p} \leq \tilde{k}_{p}, \text { or } \mathcal{S}(x)=N, \\
-\sum_{\substack{i \in U \\
x_{i}=1}} D_{i}\left(\tilde{k}_{p}\right) & \text { if } \mathcal{S}(x) \not \supset p, \\
0 & \text { otherwise. }
\end{array}\right.
$$

Note that the worst activity level a victim $i \in U$ can face is $\tilde{k}_{p}$. Indeed, if a victim $i \in U$ chooses to be active in a coalition that does not contain the polluter, it expects that the remaining agents reorganize themselves into the worst configuration for it. On the other hand, by construction of the game the polluter is encouraged to be the only active agent among the remaining ones. Therefore the polluter chooses the activity level $\tilde{k}_{p}$ which maximizes its private benefit.

Proposition 3.1. For any mapping of rights $\phi_{\tilde{k}_{p}} \in \tilde{\Phi}$, for any social cost problem $P \in \mathcal{P}$, the corresponding multi-choice social cost game $v_{\hat{\phi}_{p}, P}$ has a non-empty core.

The proof is provided in appendix A. Proposition 3.1 ensures that it is possible to reach a stable agreement whatever the threshold $\tilde{k}_{p}$ imposed by the law on the activity of the polluter, and regardless the social cost problem. According to the definition of the core, even if the law imposes a threshold on the polluter activity that is lower than the optimal level for the whole society, agents will participate in the negotiation through a participation profile $\left(1, \ldots, 1, k_{p}\right) \in L$.

Note that this result is strongly related to the pessimistic hypothesis regarding the behavior of the remaining agents and the negative externality the polluter generates. Any mapping 
of rights in $\tilde{\Phi}$ allows the polluter to generate an activity. By expecting the worst case, victim $i \in U$ anticipates that the polluter will run its activity alone if it deviates from any agreement reached by a coalition containing $p$. The negative externality generated by the polluter typically reduces the incentives for victims to free-ride.

Proposition 3.2. The only mapping of rights in $\Phi$ that satisfies core compatibility and noveto power for a victim is $\phi_{l_{p}} \in \tilde{\Phi} \subseteq \Phi$.

The proof is provided in appendix B. Though all mappings of rights in $\tilde{\Phi}$ satisfy core compatibility, only one satisfies no-veto power for a victim. This confirm the result from Gonzalez et al. (2019) that identify only one possibility to conciliate the efficiency thesis with the requirement that no victim can veto an agreement reached by the rest of the society. Therefore, the only possibility to moderate the power of isolated victims while imposing core compatibility is $\phi_{l_{p}} \in \tilde{\Phi}$.

\subsection{Enhancing the role of victims:}

Here we argue that agent $i \in N$ has power in negotiations since there exists at least one participation profile $x \in L$ such that $\phi\left(x_{-i}, 0_{i}\right)=0$. In other words, an agent $i$ has power if it can veto at least one agreement. While mappings of rights in $\tilde{\Phi}$ mainly rely on the involvement of the polluter, they may give power to victims in the negotiation only for participation profiles where the polluter decides to implement an activity level beyond the regulated level $\tilde{k}_{p}$.

In order to give more power to victims, we introduce a new family of mappings which assigns the rights to subset of victims. Notice that conditions $(\mathrm{C} 1)-(\mathrm{C} 4)$ do not precise the behavior of a mapping of rights when the polluter becomes active while a subset of victims $S \subseteq U$ has already received the rights. It is possible that active victims loose the rights or retain them. From that point, we should accurately describe the behavior of a mapping which assigns the rights to a subset of victims whenever the polluter becomes active.

We introduce the subset of mappings which allows only subsets of victims and whole society to negotiate. Let $\emptyset \neq S \subseteq U$ be any group of victims, we define $\phi_{S}^{u} \in \Phi$ as follows :

$$
\phi_{S}^{u}(x)= \begin{cases}1 & \text { if } S \subseteq \mathcal{S}(x) \subseteq U \text { or } \mathcal{S}(x)=N \\ 0 & \text { otherwise }\end{cases}
$$

Also, we introduce the mapping that gives the rights to the grand coalition only, denoted by $\phi^{\prime u} \in \Phi:$

$$
\phi^{\prime u}(x)=1 \text { if } \mathcal{S}(x)=N, \quad \phi^{\prime u}(x)=0 \text { otherwise. }
$$

We denote by $\Phi^{u} \subseteq \Phi$ the family of mappings which assigns the rights to victims or to the whole society exclusively.

Given a social cost problem $P \in \mathcal{P}$, and whatever $\phi^{u} \in \Phi^{u}$, the multi-choice cooperative game $v_{\phi^{u}, P} \in M C$ is as follows:

$$
v_{\phi^{u}, P}(x)=B_{p}\left(k_{x}^{*}\right)-\sum_{i \in U} D_{i}\left(k_{x}^{*}\right) \text { if } \mathcal{S}(x)=N, \quad v_{\phi^{u}, P}(x)=0 \text { otherwise. }
$$

Since the polluter can only negotiate with the whole set of victims, the worth of each participation profile $x \in L$ where $\mathcal{S}(x) \subsetneq N$ is $v_{\phi_{S}^{u}}^{u} P(x)=0$. Finally, only participation profiles 
$x \in L$ in which each agent is active, $\mathcal{S}(x)=N$, may have a positive worth $v_{\phi_{S}^{u}, P}(x) \geq 0$. From this, it is straightforward that each mappings of rights $\phi^{u} \in \Phi^{u}$ satisfies core compatibility. It should be noticed that this family of mapping of rights fully captures the idea behind the polluter-pays principle. Indeed, such assignment of rights always considers the polluter liable for the harmful damage. Further, there does not exist any configuration in which the polluter can be active except by contracting with the whole society and thus by compensating each victim.

By the use of propositions 3.1 and 3.2, we are now able to introduce our main result regarding core compatibility.

Theorem 3.3. The only mappings of rights in $\Phi$ that satisfy core compatibility are the mappings in $\Phi^{u} \cup \tilde{\Phi}$.

The proof is provided in appendix C. The intuition of this result is as follows. First, it is clear that any mapping of rights in $\Phi^{u}$ satisfies core compatibility. By proposition 3.1 we know that any $\phi_{\tilde{k}_{p}} \in \tilde{\Phi}$ is core compatible. Then, we use proposition 3.2 to show that if a mapping of right $\phi \in \Phi \backslash \tilde{\Phi}$ is core compatible, this forces $\phi \in \Phi^{u}$.

This result aligns with the existing cooperative game literature which has analyzed the Coase theorem. It highlights the impossibility to satisfies both efficiency and neutrality theses.

Theorem 3.4. There is no mapping of rights in $\Phi$ that satisfies Kaldor-Hicks core compatibility and no veto power for a victim.

Theorem 3.4. Notice that any mapping of rights $\phi^{u} \in \Phi^{u}$ satisfies Kaldor-Hicks core compatibility. But it does not satisfy no veto power for a victim.

By proposition 3.2, the only mapping of rights that satisfies core compatibility and no veto power for a victim is $\phi_{l_{p}} \in \tilde{\Phi}$. By definition of the corresponding game $v_{\phi_{l_{p}}, P}$ given by (5), it holds that:

$$
v_{\phi_{l_{p}}, P}\left(0_{-p}, k_{p}\right)=B_{p}\left(k_{p}\right), \text { and } v_{\phi_{l_{p}}, P}\left(1_{-p}, k_{p}\right)=B_{p}\left(k_{x}^{*}\right)-\sum_{i \in U} D_{i}\left(k_{x}^{*}\right), \text { where } k_{p} \in L_{p} .
$$

For each $k_{p} \in L_{p}$ we have $v_{\phi_{l_{p}}, P}\left(0_{-p}, k_{p}\right) \geq v_{\phi_{l_{p}}, P}\left(1_{-p}, k_{p}\right)$. However, the core constraints impose for each $k_{p} \in L_{p}$,

$$
\psi_{p, k_{p}} \geq v_{\phi_{l_{p}}, P}\left(0_{-p}, k_{p}\right), \text { and } \sum_{i \in U} \psi_{i, 1}+\psi_{p, k_{p}}=v_{\phi_{l_{p}}, P}\left(1_{-p}, k_{p}\right) .
$$

We can easily construct a social cost problem $P \in \mathcal{P}$ such that $v_{\phi_{l_{p}}, P}\left(0_{-p}, k_{p}\right)>v_{\phi_{l_{p}}, P}\left(1_{-p}, k_{p}\right) \geq$ 0 . In this case we have:

$$
\forall k_{p} \in L_{p}, \quad \psi_{p, k_{p}}>\sum_{i \in U} \psi_{i, 1}+\psi_{p, k_{p}} \geq 0
$$

This implies that:

$$
\sum_{i \in U} \psi_{i, 1}<0
$$

Therefore, the mapping of rights $\phi_{l_{p}} \in \tilde{\Phi}$ does not satisfy Kaldor-Hicks core compatibility. This concludes the proof. 


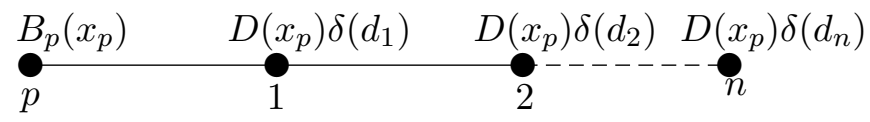

Figure 1: Spatial social cost problem.

According to proposition 3.4 it is not possible to conciliate the efficiency thesis while imposing full compensation and no veto power for a victim.

\section{Illustration with a spatial case}

Theorem 3.3 states that the only core compatible mappings of rights lies in $\Phi^{u} \cup \tilde{\Phi}$. In this section, we illustrates this theorem through an example and provide a core payoff vector. Assume that agents are located on a line. The polluter is the unique source of the pollution. For convenience, we consider the polluter is located at one end of the line and each victim $i \in U$ has the same damage function $D$. The only difference between victims comes from their distance $d_{i} \in \mathbb{R}_{+}$from the polluter. Hence, the damage suffered from the pollution at given location depends both on the activity level of the polluter $k_{p} \in L_{p}$ and on the distance $d_{i}$ from the polluter. The higher the activity level of the polluter the higher the impact on the victim $i \in U$. On the other hand, the further away a victim $i$ is from the polluter, the less it suffers from the pollution. This is captured by the decreasing function $\delta: \mathbb{R}_{+} \rightarrow \mathbb{R}_{+}$. Figure 1 provides a spatial representation when at least three agents are involved in a social cost problem.

Consider a social cost problem close to the one presented in Example 2, and the mapping of rights $\phi_{\tilde{k}_{p}} \in \tilde{\Phi}$. Let say that the regulated level is $\tilde{k}_{p}=2$, so that we write $\phi_{\tilde{k}_{p}}=\phi_{2}$. Let $N:=\{1,2, p\}$ be the set of agents, $L_{p}:=\{0,1,2,3,4\}$ is the action set of the polluter, $L_{1}=L_{2}=\{0,1\}$, and

$$
B_{p}\left(x_{p}\right)=4 \sqrt{x_{p}}, \quad D\left(x_{p}\right)=2 x_{p} .
$$

Assume that $\delta\left(d_{1}\right)=6 / 10$ and $\delta\left(d_{2}\right)=3 / 10$. The conditions for a payoff vector $\psi \in \mathbb{R}^{M}$ to be in the core of the multi-choice game $v_{\phi_{2}, P} \in M C$ are as follows:

$$
\begin{aligned}
\psi_{1,1}+\psi_{2,1}+\psi_{p, k} & =v_{\phi_{2}, P}(1,1, k)=2.2, \quad \text { for each } k \in L_{p} \backslash\{0\} \\
\psi_{1,1}+\psi_{p, 1} \geq v_{\phi_{2}, P}(1,0,1) & =2.8 \\
\psi_{1,1}+\psi_{p, 2} \geq v_{\phi_{2}, P}(1,0,2) & =3.26 \\
\psi_{2,1}+\psi_{p, 1} \geq v_{\phi_{2}, P}(0,1,1) & =3.4 \\
\psi_{2,1}+\psi_{p, 1} \geq v_{\phi_{2}, P}(0,1,1) & =4.45 \\
\psi_{1,1}+\psi_{1,2} \geq v_{\phi_{2}, P}(1,1,0) & =-3.6 \\
\psi_{p, 1} \geq v_{\phi_{2}, P}(0,0,1) & =4 \\
\psi_{p, 2} \geq v_{\phi_{2}, P}(0,0,2) & =5.6
\end{aligned}
$$

We can remark that for each participation profile such that $\mathcal{S}(x)=N$, the optimal activity level is $k_{x}^{*}=1$. Thus, a payoff vector in the core assigns the same value for each participation 
level to the polluter. For instance, one can verify that the payoff-vector $\psi \in \mathbb{R}^{M}$ such that $\psi_{1,1}=-2.3, \psi_{2,1}=-1.2$, and for each $x_{p} \in L_{p} \backslash\{0\} \psi_{p, x_{p}}=5.7$ satisfies all the above inequalities defining the core. This payoff-vector indicates that victims reach an agreement with the polluter where the activity level is $k_{x}^{*}=1$. Victims compensate the polluter by -2.3 and -1.2 for reducing its activity level.

By contrast, consider the mapping of rights $\phi^{u^{\prime}} \in \Phi^{u}$, and the corresponding multi-choice game $v_{\phi^{u^{\prime}}, P} \in M C$ as defined in section 3.2. The conditions for a payoff vector $\psi \in \mathbb{R}^{M}$ to be in the core of the multi-choice game $v_{\phi^{u^{\prime}}, P} \in M C$ are as follows:

$$
\begin{aligned}
\psi_{i, 1} & \geq 0, \quad \text { for } i=1,2 \\
\psi_{p, k} & \geq 0, \quad \text { for } k=1, \ldots, 4 \\
\psi_{1,1}+\psi_{2,1}+\psi_{p, k} & =2.2=v_{\phi^{u^{\prime}}, P}(1,1, k), \quad \text { for each } k \in L_{p} \backslash\{0\} .
\end{aligned}
$$

In this case, the equal payoff vector $\psi \in \mathbb{R}^{M}$ such that $\psi_{1,1}=\psi_{2,1}=\psi_{p, k}=v_{\phi^{u^{\prime}}, P}(1,1, k) / 3$ lies in the core.

\section{Concluding remarks}

In this paper, we have analyzed the legal structure ruling negotiations among a finite set of agents when the activity of one agent is harmful for the rest of the society. Examples include any situations where agents with conflicting interests aim to negotiate compensation for the damage.

We contribute to the literature devoted to the Coase theorem by deriving a multi-choice cooperative game from a social cost problem and a liability rule (defined by mappings of rights). We identify two specific families of liability rules that enable to satisfy the efficiency thesis formalized in terms of non-emptiness of the core. We show that any regulation that imposes a quota on the activity level of the polluter is compatible with the efficiency thesis, provided that the whole set of potential victims can negotiate an agreement with the polluter. In the same way we show that any mapping that exclusively assigns the rights to a set of victim satisfies the efficiency thesis. However, we show that it is not possible to conciliate the efficiency thesis while requiring full compensation property and no veto power for a victim. Let us mention that our construction of the multi-choice game relies on the assumption that agents form pessimistic expectations regarding the behavior of the remaining agents. Under other assumptions, a multi-choice game associated with the mapping of rights which considers quotas on the polluter's activity level may have an empty core. Indeed, the pessimistic expectation assumption restricts the incentives for isolate victims to benefit from an agreement reached by the rest of the society without contributing, that is free-riding.

\section{Appendix A: Proof of proposition 3.1}

Proposition 3.1. To prove proposition 3.1, remark that the conditions to have a balanced collection $\mathcal{B}$ can be rewritten as follows. A collection $\mathcal{B}$ is balanced if there exists positive 
coefficients $\mu(x), x \in \mathcal{B}$, such that:

$$
\begin{aligned}
\forall i \in U, \sum_{\substack{x \in \mathcal{B} \\
x_{i}=1}} \mu(x)=l_{p}, \\
\forall k=1, \ldots, l_{p}, \sum_{\substack{x \in \mathcal{B} \\
x_{p}=k}} \mu(x)=1 .
\end{aligned}
$$

Thus, a multi-choice game $v_{\phi, P}$ derived from a mapping of rights $\phi \in \Phi$ and a social cost problem $P \in \mathcal{P}$, is balanced if for every balanced collection $\mathcal{B}$ of elements of $L \backslash\{(0, \ldots, 0)\}$ it holds

$$
\sum_{x \in \mathcal{B}} \mu(x) v_{\phi, P}(x) \leq \sum_{k_{p}=1}^{l_{p}} v_{\phi, P}\left(1_{-p}, k_{p}\right) .
$$

This remark allows us to derive the following intermediary result regarding balanced collection.

Lemma 5.1. Let $\mathcal{B}$ be a balanced collection and $\mu(x), x \in \mathcal{B}$, the associated balancing weights. Then, the following holds:

$$
\forall i \in U, \quad \sum_{\substack{x \in \mathcal{B} \\
x_{i}=1, x_{p}=0}} \mu(x)=\sum_{k=1}^{l_{p}} \sum_{\begin{array}{c}
x \in \mathcal{B} \\
x_{i}=0, \\
x_{p}=k
\end{array}} \mu(x) .
$$

We interpret this lemma through time allocation in the same way as for balanced collection. This lemma simply indicates that the amount of time each victim spends in coalitions where the polluter is not active is equal to the total amount of time the polluter spends in coalitions where the victim is not active. This constitutes the main argument in order to prove proposition 3.1. Indeed, according to this lemma we can consider a victim in terms of participation profiles in which it is not active but the polluter is.

Proof. By definition of a balanced collection it holds that

$$
\begin{aligned}
\forall i \in U, l_{p}= & \sum_{\substack{x \in \mathcal{B} \\
x_{i}=1}} \mu(x) \\
= & \sum_{\substack{x \in \mathcal{B} \\
x_{i}=1, x_{p}=0}} \mu(x)+\sum_{\substack{x \in \mathcal{B} \\
x_{i}=1, x_{p}=1}} \mu(x)+\ldots+\sum_{\substack{x \in \mathcal{B} \\
x_{i}=1, x_{p}=l_{p}}} \mu(x) \\
= & \sum_{\substack{x \in \mathcal{B} \\
x_{i}=1, x_{p}=0}} \mu(x)+\sum_{\substack{l_{p} \\
k=1}} \sum_{\substack{x \in \mathcal{B} \\
x_{i}=1, x_{p}=k}} \mu(x)
\end{aligned}
$$

So we can write

$$
\sum_{\substack{x \in \mathcal{B} \\ x_{i}=1 \\ x_{p}=0}} \mu(x)=l_{p}-\sum_{k=1}^{l_{p}} \sum_{\substack{x \in \mathcal{B} \\ x_{i}=1 \\ x_{p}=k}} \mu(x)
$$


By definition, for each $k=1, \ldots, l_{p}$ we have

$$
\sum_{\substack{x \in \mathcal{B} \\ x_{i}=1 \\ x_{p}=k}} \mu(x)=1-\sum_{\substack{x \in \mathcal{B} \\ x_{i}=0 \\ x_{p}=k}} \mu(x)
$$

By substituting (9) in (8) we obtain

$$
\begin{gathered}
l_{p}=\sum_{\substack{x \in \mathcal{B} \\
x_{i}=1 \\
x_{p}=0}} \mu(x)+\sum_{k=1}^{l_{p}}\left(1-\sum_{\substack{x \in \mathcal{B} \\
x_{i}=0 \\
x_{p}=k}} \mu(x)\right) \\
=\sum_{\substack{x \in \mathcal{B} \\
x_{i}=1 \\
x_{p}=0}} \mu(x)+l_{p}-\sum_{k=1}^{l_{p}} \sum_{\substack{x \in \mathcal{B} \\
x_{i}=0 \\
x_{p}=k}} \mu(x)
\end{gathered}
$$

and so

$$
\sum_{\substack{x \in \mathcal{B} \\ x_{i}=1 \\ x_{p}=0}} \mu(x)=\sum_{k=1}^{l_{p}} \sum_{\substack{x \in \mathcal{B} \\ x_{i}=0 \\ x_{p}=k}} \mu(x) .
$$

In order to prove proposition 3.1, consider the modified Bondareva-Shapley theorem for multi-choice settings (proposition 2.1). We have to show that for any mapping of rights $\phi_{\tilde{k}_{p}} \in \tilde{\Phi}$ and for any social cost problem $P \in \mathcal{P}$, the corresponding game is balanced. Let $\mathcal{B}$ be any balanced collection of elements of $L \backslash\{(0, \ldots, 0)\}$ and $\mu(x), x \in \mathcal{B}$, the associated balancing weights. First of all, notice that by definition of $v_{\tilde{k}_{p}, P}$ given by (5) each participation profile $x \in L$ such that $p \in \mathcal{S}(x) \subsetneq N$ and $x_{p}>\tilde{k}_{p}$ we have: $v_{\phi_{\tilde{k}_{p}}, P}(x)=0$. Therefore, we have to consider three types of participation profile: $x \in L$ such that $0<x_{p} \leq \tilde{k}_{p}, x \in L$ such that $x_{p}>\tilde{k}_{p}$ and $\mathcal{S}(x)=N$, and finally $x \in L$ such that $x_{p}=0$. From this point we have:

$$
\begin{aligned}
\sum_{x \in \mathcal{B}} \mu(x) v_{\phi_{\tilde{k}_{p}}, P}(x) & =\sum_{k=1}^{\tilde{k}_{p}} \sum_{\substack{x \in \mathcal{B} \\
x_{p}=k}} v_{\phi_{\tilde{k}_{p}}, P}(x)+\sum_{k^{\prime}=\tilde{k}_{p}+1}^{l_{p}} \sum_{\begin{array}{c}
x \in \mathcal{B} \\
x_{p}=k^{\prime} \\
\mathcal{S}(x)=N
\end{array}} \mu(x) v_{\tilde{k}_{p}, P}(x) \\
& +\sum_{\substack{x \in \mathcal{B} \\
x_{p}=0}} \mu(x) v_{\phi_{\tilde{k}_{p}}, P}(x) .
\end{aligned}
$$


Using (5) which gives the corresponding worth of $v_{\phi_{\tilde{k}_{p}}, P}$, we have:

$$
\begin{aligned}
\sum_{x \in \mathcal{B}} \mu(x) v_{\phi_{\tilde{k}_{p}}, P}(x) & =\sum_{k=1}^{\tilde{k}_{p}} \sum_{\substack{x \in \mathcal{B} \\
x_{p}=k}} \mu(x)\left(B_{p}\left(k_{x}^{*}\right)-\sum_{i \in U} D_{i}\left(k_{x}^{*}\right)\right) \\
& +\sum_{x_{i}=1}^{l_{p}} \sum_{\substack{x \in \mathcal{B} \\
k^{\prime}=\tilde{k}_{p}+1 \\
x_{p}=k^{\prime} \\
\mathcal{S}(x)=N}} \mu(x)\left(B_{p}\left(k_{x}^{\prime *}\right)-\sum_{i \in U} D_{i}\left(k_{x}^{\prime *}\right)\right) \\
& -\sum_{\substack{x \in \mathcal{B} \\
x_{p=0}}} \mu(x)\left(\sum_{i \in U} D_{i}\left(\tilde{k}_{p}\right)\right) .
\end{aligned}
$$

Let us focus on the last part of the right hand-side of equation (12). In other words, we focus on the worth of participation profiles in which the polluter is inactive. It holds that

$$
-\sum_{\substack{x \in \mathcal{B} \\ x_{p}=0}} \mu(x)\left(\sum_{\substack{i \in U \\ x_{i}=1}} D_{i}\left(\tilde{k}_{p}\right)\right)=-\sum_{i \in U} D_{i}\left(\tilde{k}_{p}\right)\left(\sum_{\substack{x \in \mathcal{B} \\ x_{i}=1 \\ x_{p}=0}} \mu(x)\right) .
$$

Using lemma 5.1 we obtain:

$$
\begin{aligned}
-\sum_{i \in U} D_{i}\left(\tilde{k}_{p}\right)\left(\sum_{\substack{x \in \mathcal{B} \\
x_{i}=1 \\
x_{p}=0}} \mu(x)\right) & =-\sum_{i \in U} D_{i}\left(\tilde{k}_{p}\right)\left(\sum_{k=1}^{l_{p}} \sum_{\substack{x \in \mathcal{B} \\
x_{i}=0 \\
x_{p}=k}} \mu(x)\right) \\
& =-\sum_{k=1}^{l_{p}} \sum_{\substack{x \in \mathcal{B} \\
x_{p}=k}} \mu(x)\left(\sum_{\substack{i \in U \\
x_{i}=0}} D_{i}\left(\tilde{k}_{p}\right)\right) .
\end{aligned}
$$

Therefore, by lemma 5.1 we can replace the sum of balancing weights for participation profiles in which a victim is active while the polluter is inactive by the sum of balancing weights for participation profiles $x \in \mathcal{B}$ in which the victim is inactive while the polluter is active. By 
replacing 13$)$ in 12 we obtain:

$$
\begin{aligned}
& \sum_{x \in \mathcal{B}} \mu(x) v_{\phi_{\tilde{k}_{p}}, P}(x)=\sum_{k=1}^{\tilde{k}_{p}} \sum_{\substack{x \in \mathcal{B} \\
x_{p}=k}} \mu(x)\left(B_{p}\left(k_{x}^{*}\right)-\sum_{\substack{i \in U \\
x_{i}=1}} D_{i}\left(k_{x}^{*}\right)\right)+\sum_{\substack{k^{\prime}=\tilde{k}_{p}+1 \\
k_{p}}} \sum_{\substack{x \in \mathcal{B} \\
x_{p}=k^{\prime} \\
\mathcal{S}(x)=N}} \mu(x)\left(B_{p}\left(k_{x}^{\prime *}\right)-\sum_{i \in U} D_{i}\left(k_{x}^{\prime *}\right)\right) \\
& -\sum_{k=1}^{l_{p}} \sum_{\substack{x \in \mathcal{B} \\
x_{p}=k}} \mu(x)\left(\sum_{\substack{i \in U \\
x_{i}=0}} D_{i}\left(\tilde{k}_{p}\right)\right) \\
& =\sum_{k=1}^{\tilde{k}_{p}} \sum_{\substack{x \in \mathcal{B} \\
x_{p}=k}} \mu(x)\left(B_{p}\left(k_{x}^{*}\right)-\sum_{\substack{i \in U \\
x_{i}=1}} D_{i}\left(k_{x}^{*}\right)\right)+\sum_{\substack{l_{p} \\
k^{\prime}=\tilde{k}_{p}+1}} \sum_{\substack{x \in \mathcal{B} \\
x_{p}=k^{\prime} \\
\mathcal{S}(x)=N}} \mu(x)\left(B_{p}\left(k_{x}^{*}\right)-\sum_{i \in U} D_{i}\left(k_{x}^{*}\right)\right) \\
& -\sum_{k=1}^{\tilde{k}_{p}} \sum_{\substack{x \in \mathcal{B} \\
x_{p}=k}} \mu(x)\left(\sum_{\substack{i \in U \\
x_{i}=0}} D_{i}\left(\tilde{k}_{p}\right)\right)-\sum_{k^{\prime}=\tilde{k}_{p}+1}^{l_{p}} \sum_{\substack{x \in \mathcal{B} \\
x_{p}=k^{\prime}}} \mu(x)\left(\sum_{\substack{i \in U \\
x_{i}=0}} D_{i}\left(\tilde{k}_{p}\right)\right) .
\end{aligned}
$$

Notice that for each $x \in \mathcal{B}$ such that $x_{p}=k_{p} \leq \tilde{k}_{p}$, the optimal level negotiated by agents given the participation profile $x$ must be lower than or equal to the regulated level: $k_{x}^{*} \leq \tilde{k}_{p}$, where $k_{x}^{*} \in K_{x}^{*}$. Since for each $i \in U, D_{i}$ is a non-decreasing function, we have $D_{i}\left(\tilde{k}_{p}\right) \geq$ $D_{i}\left(k_{x}^{*}\right)$. Therefore we obtain:

$$
\begin{aligned}
\sum_{x \in \mathcal{B}} \mu(x) v_{\phi_{\tilde{k}_{p}}, P}(x) \leq & \sum_{k=1}^{\tilde{k}_{p}} \sum_{\substack{x \in \mathcal{B} \\
x_{p}=k}} \mu(x)\left(B_{p}\left(k_{x}^{*}\right)-\sum_{\substack{i \in U \\
x_{i}=1}} D_{i}\left(k_{x}^{*}\right)\right)+\sum_{k^{\prime}=\tilde{k}_{p}+1} \sum_{\substack{x \in \mathcal{B} \\
x_{p}=k^{\prime} \\
\mathcal{S}(x)=N}} \mu(x)\left(B_{p}\left(k_{x}^{\prime *}\right)-\sum_{i \in U} D_{i}\left(k_{x}^{\prime *}\right)\right) \\
& -\sum_{k=1}^{\tilde{k}_{p}} \sum_{\substack{x \in \mathcal{B} \\
x_{p}=k}} \mu(x)\left(\sum_{\substack{i \in U \\
x_{i}=0}} D_{i}\left(k_{x}^{*}\right)\right)-\sum_{k^{\prime}=\tilde{k}_{p}+1}^{l_{p}} \sum_{\substack{x \in \mathcal{B} \\
x_{p}=k^{\prime}}} \mu(x)\left(\sum_{\substack{i \in U \\
x_{i}=0}} D_{i}\left(\tilde{k}_{p}\right)\right) .
\end{aligned}
$$

Remark that,

$$
-\sum_{k^{\prime}=\tilde{k}_{p}+1}^{l_{p}} \sum_{\substack{x \in \mathcal{B} \\ x_{p}=k^{\prime}}} \mu(x)\left(\sum_{\substack{i \in U \\ x_{i}=0}} D_{i}\left(\tilde{k}_{p}\right)\right) \leq 0 .
$$


So we have:

$$
\begin{aligned}
\sum_{x \in \mathcal{B}} \mu(x) v_{\phi_{\tilde{k}_{p}}, P}(x) & \leq \sum_{k=1}^{\tilde{k}_{p}} \sum_{\substack{x \in \mathcal{B} \\
x_{p}=k}} \mu(x)\left(B_{p}\left(k_{x}^{*}\right)-\sum_{\substack{i \in U \\
x_{i}=1}} D_{i}\left(k_{x}^{*}\right)\right)-\sum_{k=1} \sum_{\substack{x \in \mathcal{B} \\
x_{p}=k}} \mu(x)\left(\sum_{\substack{i \in U \\
x_{i}=0}} D_{i}\left(k_{x}^{*}\right)\right) \\
& +\sum_{k^{\prime}=\tilde{k}_{p}+1}^{l_{p}} \sum_{\substack{x \in \mathcal{B} \\
x_{p}=k^{\prime} \\
\mathcal{S}(x)=N}} \mu(x)\left(B_{p}\left(k_{x}^{\prime *}\right)-\sum_{i \in U} D_{i}\left(k_{x}^{\prime *}\right)\right) .
\end{aligned}
$$

For participation profiles $x \in \mathcal{B}$ such that $x_{p} \leq \tilde{k}_{p}$, the corresponding optimal activity level $k_{x}^{*}$ considers only active victims, but we insert this activity level $k_{x}^{*}$ for inactive victims rather than $\tilde{k}_{p}$. Hence, every agents face the activity level $k_{x}^{*}$ decided by the active agents in $x \in \mathcal{B}$.

Therefore we obtain:

$$
\sum_{x \in \mathcal{B}} \mu(x) v_{\phi_{\tilde{k}_{p}}, P}(x) \leq \sum_{k=1}^{\tilde{k}_{p}} \sum_{\substack{x \in \mathcal{B} \\ x_{p}=k}} \mu(x)\left(B_{p}\left(k_{x}^{*}\right)-\sum_{i \in U} D_{i}\left(k_{x}^{*}\right)\right)+\sum_{\substack{k^{\prime}=\tilde{k}_{p}+1 \\ l_{p}}} \sum_{\substack{x \in \mathcal{B} \\ x_{p}=k^{\prime} \\ \mathcal{S}(x)=N}} \mu(x)\left(B_{p}\left(k_{x}^{\prime *}\right)-\sum_{i \in U} D_{i}\left(k_{x}^{\prime *}\right)\right) .
$$

However, the activity level $k_{x}^{*}$ is not necessarily optimal when we consider all agents. Therefore, we have

$$
\sum_{x \in \mathcal{B}} \mu(x) v_{\phi_{\tilde{k}_{p}}, P}(x) \leq \sum_{k=1}^{\tilde{k}_{p}} \max _{h \in[[0, k]]}\left(B_{p}(h)-\sum_{i \in U} D_{i}(h)\right)\left(\sum_{\substack{x \in \mathcal{B} \\
x_{p}=k}} \mu(x)\right)+\sum_{\substack{k^{\prime}=\tilde{k}_{p}+1 \\
k_{p}}} \sum_{\begin{array}{c}
x \in \mathcal{B} \\
x_{p}=k^{\prime} \\
\mathcal{S}(x)=N
\end{array}} \mu(x)\left(B_{p}\left(k_{x}^{\prime *}\right)-\sum_{i \in U} D_{i}\left(k_{x}^{\prime *}\right)\right) .
$$

Recall that, by definition of $v_{\tilde{k}_{p}, P}$, for each $x \in L$ such that $x_{p}=k_{p} \leq \tilde{k}_{p}$ :

$$
\max _{h \in\left[\left[0, k_{p}\right]\right]}\left(B_{p}(h)-\sum_{i \in U} D_{i}(h)\right)=v_{\phi_{\tilde{k}_{p}}, P}\left(1_{-p}, k_{p}\right),
$$

and for each $x \in L$ such that $x_{p}=k_{p}^{\prime}>\tilde{k}_{p}$, with $\mathcal{S}(x)=N$ :

$$
\left(B_{p}\left(k_{x}^{\prime *}\right)-\sum_{i \in U} D_{i}\left(k_{x}^{\prime *}\right)\right)=v_{\phi_{\tilde{k}_{p}}, P}\left(1_{-p}, k_{p}^{\prime}\right) .
$$

Therefore, we have:

$$
\sum_{x \in \mathcal{B}} \mu(x) v_{\phi_{\tilde{k}_{p}}, P}(x) \leq \sum_{k=1}^{\tilde{k}_{p}} v_{\phi_{\tilde{k}_{p}}, P}\left(1_{-p}, k_{p}\right)\left(\sum_{\substack{x \in \mathcal{B} \\ x_{p}=k}} \mu(x)\right)+\sum_{\substack{k^{\prime}=\tilde{k}_{p}+1 \\ l_{p}}} \sum_{\substack{x \in \mathcal{B} \\ x_{p}=k^{\prime} \\ \mathcal{S}(x)=N}} \mu(x) v_{\phi_{\tilde{k}_{p}}, P}\left(1_{-p}, k_{p}^{\prime}\right) .
$$


Finally, by definition of the balanced collection, for each $k \in L_{p}, \sum_{\substack{x \in \mathcal{B} \\ x_{p}=k}} \mu(x)=1$. Then we obtain:

$$
\sum_{x \in \mathcal{B}} \mu(x) v_{\tilde{k}_{p}, P}(x) \leq \sum_{k=1}^{\tilde{k}_{p}} v_{\phi_{\tilde{k}_{p}}, P}\left(1_{-p}, k_{p}\right)+\sum_{k^{\prime}=\tilde{k}_{p}+1}^{l_{p}} v_{\tilde{k}_{p}, P}\left(1_{-p}, k_{p}^{\prime}\right) .
$$

Therefore, by equation (7) we conclude that the multi-choice game $v_{\phi_{\tilde{k}_{p}}, P}$ is balanced. By proposition 2.1. $\mathcal{C}\left(v_{\phi_{\tilde{k}_{p}}, P}\right) \neq \emptyset$.

\section{Appendix B: Proof of proposition 3.2}

Proposition 3.2. By proposition 3.1, $\phi_{l_{p}} \in \tilde{\Phi}$ satisfies core compatibility. By definition of $\phi_{l_{p}}$ and conditions (C2) and (C3) defining mappings of rights, for any victim $i \in U$, for any $x \in L$ such that $\mathcal{S}(x)=N \backslash\{i\}, \phi^{p}\left(1_{-i, p}, 0_{i}, x_{p}\right)=1$. Hence, $\phi_{l_{p}}$ satisfies no-veto power for a victim. But this is not the case for others mapping of rights in $\tilde{\Phi}$. Indeed, for any $\phi_{\tilde{k}_{p}} \in \tilde{\Phi} \backslash\left\{\phi_{l_{p}}\right\}$ defined with respect to any regulated level $\tilde{k}_{p}<l_{p}$, we must have $\phi_{\tilde{k}_{p}}\left(1_{-i, p}, 0_{i}, l_{p}\right)=0$. So, $\phi_{\tilde{k}_{p}} \in \tilde{\Phi} \backslash\left\{\phi_{l_{p}}\right\}$ violates no-veto power for a victim.

To show that $\phi_{l_{p}}$ is the only mapping of rights satisfying core compatibility and no veto power, consider any mapping of rights $\phi \in \Phi \backslash \tilde{\Phi}$ that satisfy no-veto power. By (C3), it must be the case that for each victim $i \in U, \phi\left(1_{-i, p}, 0_{i}, x_{p}\right)=1$, where $\mathcal{S}(x)=N \backslash\{i\}$. Combined with (C4) it implies that for each victim $i \in U, \phi\left(0_{-i}, 1_{i}\right)=0$. Furthermore, we have $\phi\left(0_{-p}, x_{p}\right)=0$, otherwise $\phi \in \tilde{\Phi}$. Following this, there exists only two types of mapping of rights for $\phi \in \Phi \backslash \tilde{\Phi}$ :

(a) for each $i \in N$, for each $x \in L$ such that $\mathcal{S}(x)=N \backslash\{i\}, \phi\left(x_{-i}, 0_{i}\right)=1$ and $\phi\left(1_{-p}, 0_{p}\right)=$ 1 ,

(b) for each $i \in N \backslash\{p\}$, for each $x \in L$ such that $\mathcal{S}(x)=N \backslash\{i\}, \phi\left(x_{-i}, 0_{i}\right)=1$ and $\phi\left(1_{-p}, 0_{p}\right)=0$.

To prove that $\phi \in \Phi \backslash \tilde{\Phi}$ does not satisfy core compatibility consider the following social cost problem.

The set of agents is $N:=\{1,2, p\}$. For each agent, the action sets are $L_{1}=L_{2}=\{0,1\}$, $L_{p}=\{0,1,2,3\}$. The benefit function and damage function for each agent are such that, for each activity level $k \in L_{p}$ :

$$
B_{p}(k)=7 \sqrt{k}, \quad D_{1}(k)=3 k, \quad D_{2}(k)=2 k .
$$

Given the social cost problem $P \in \mathcal{P}$ and the mapping of rights $\phi^{(a)}, \phi^{(b)}$, we construct the multi-choice game $\left(N, L, v_{\phi, P}\right)$. Notice that for both types of mapping of rights we have: $v_{\phi, P}(1,1,0)=0$, for each $k \in L_{p}, v_{\phi, P}(0,0, k)=0$ and

$$
\begin{array}{rrr}
v_{\phi, P}(1,0,1)=4, & v_{\phi, P}(1,0,2)=4, & v_{\phi, P}(1,0,3)=4, \\
v_{\phi, P}(0,1,1)=5, & v_{\phi, P}(0,1,2)=5.89, & v_{\phi, P}(0,1,3)=6.12, \\
v_{\phi, P}(1,1,1)=2, & v_{\phi, P}(1,1,2)=2, & v_{\phi, P}(1,1,3)=2 .
\end{array}
$$


Consider the balanced collection

$$
\mathcal{B}:=\{(1,1,0),(1,0,1),(0,1,1),(1,0,2),(0,1,2),(1,0,3),(0,1,3)\},
$$

and the balancing weights $\mu(1,1,0)=3 / 2$ and for each $x \in \mathcal{B} \backslash\{(1,1,0)\}, \mu(x)=1 / 2$. Straightforward computation gives

$$
\sum_{x \in \mathcal{B}} \mu(x) v(x)=19.505>6=\sum_{k=1}^{3} v(1,1, k) .
$$

By proposition 2.1 the multi-choice game constructed from the mapping of rights $\phi \in \Phi \backslash \tilde{\Phi}$ and the social cost problem $P \in \mathcal{P}$ has an empty core. This concludes the proof.

\section{Appendix C: Proof of theorem 3.3}

Proof. According to proposition 3.1 each mapping of rights $\phi_{\tilde{k}_{p}} \in \tilde{\Phi}$ satisfies core compatibility. Furthermore it is obvious that any mapping of rights $\phi^{u} \in \Phi^{u}$ satisfies core compatibility. Indeed, as mentioned in section 3.2 , only participation profiles $x \in L$ such that $\mathcal{S}(x)=N$ may have positive worth. Then, for all balanced collection $\mathcal{B}$ of element of $L \backslash\{(0, \ldots, 0)\}$, it always holds that:

$$
\sum_{x \in \mathcal{B}} \mu(x) v_{\phi^{u}, P}(x) \leq \sum_{k_{p}=1}^{l_{p}} v_{\phi^{u}, P}\left(1_{-p}, k_{p}\right) .
$$

To prove that there is no other mapping of rights satisfying core compatibility consider any mapping of rights $\phi \in \Phi \backslash \tilde{\Phi}$ that satisfies core compatibility. We have to show that $\phi \in \Phi^{u}$. First, for each $x_{p} \in L_{p} \backslash\{0\}$, we have $\phi\left(0_{-p}, x_{p}\right)=0$, otherwise $\phi \in \tilde{\Phi}$. Also, by proposition 3.2 the only mapping of rights which satisfies core compatibility and no veto power for a victim is $\phi_{l_{p}} \in \tilde{\Phi}$. Denote by $J \subseteq U$ the subset of victims who do not have any veto power defined by:

$$
J=\left\{j \in U \mid \forall x_{p} \in L_{p} \backslash\{0\}, \phi\left(1_{-j, p}, 0_{j}, x_{p}\right)=1\right\} .
$$

Any mapping of rights $\phi \in \Phi \backslash \tilde{\Phi}$ that satisfies core compatibility does not satisfy no veto power for a victim, meaning $J \neq U$. Hence, there is at least one victim $i \in U$ such that $\phi\left(1_{-i, p}, 0_{i}, l_{p}\right)=0$. In other words there is at least one victim $i \in U$ who can veto the highest activity level of the polluter. However it can be the case that there exists an activity level $x_{p} \in L_{p} \backslash\left\{0, l_{p}\right\}$ such that $\phi\left(1_{-i, p}, 0_{i}, x_{p}\right)=1$.

In order to analyze such mapping of rights, we consider the following partition for the set of victims $U$. Let $\tilde{I}$ be the set of victim who possesses a veto power defined as follows,

$$
\tilde{I}:=\left\{i \in U \mid \exists x_{p}^{i} \in L_{p} \backslash\{0\} \text { such that } \forall k_{p} \geq x_{p}^{i}, \phi\left(1_{-i, p}, 0_{i}, k_{p}\right)=0\right\} .
$$

For each $i \in \tilde{I}$, let $\tilde{x}_{p}^{i} \in L_{p} \backslash\{0\}$ be the minimum activity level of the polluter such that $\phi\left(1_{-i, p}, 0_{i}, \tilde{x}_{p}^{i}\right)=0$. Either $\tilde{x}_{p}^{i}=1$, then victim $i$ has the power to veto any agreement for any activity level of the polluter; or $\tilde{x}_{p}^{i}>1$, then for each activity level of the polluter $1 \leq k_{p}<\tilde{x}_{p}^{i}$, $\phi\left(1_{-i, p}, 0_{i}, k_{p}\right)=1$. Therefore, we decompose $\tilde{I}$ into two subsets denoted by $I$ and $H$ defined as follows:

$$
\begin{aligned}
I & =\left\{i \in \tilde{I} \mid \tilde{x}_{p}^{i}>1\right\}, \\
H & =\left\{h \in \tilde{I} \mid \tilde{x}_{p}^{i}=1\right\} .
\end{aligned}
$$


$I \subseteq U$ is the subset of victims who can veto the highest participation level of the polluter but not a lower participation level $k_{p}<\tilde{x}_{p}^{i} \in L_{p} \backslash\{0\} . H \subseteq U$ is the subset of victims who can veto any participation level of the polluter. The three subsets $H, I, J$ allows us to describe every mappings of rights with respect to the veto power of victims.

1. Suppose that for each $i \in U, \phi\left(1_{-i, p}, 0_{i}, l_{p}\right)=0$, so $J=\emptyset$. We have to consider three cases:

(a) $H=U$, so for each $i \in U$, for each $x_{p} \in L_{p} \backslash\{0\}, \phi\left(1_{-i, p}, 0_{i}, x_{p}\right)=0$. Then by monotonicity of $\phi$ on $L_{-p}$, (C2) of the definition of mapping of rights, for any $S \subset U$ we have $\phi\left(1_{-S, p}, 0_{S}, x_{p}\right)=0$. Therefore for each participation profile $x \in L$ in which the polluter is active $p \in \mathcal{S}(x)$, we must have $\phi(x)=0$. Therefore, $\phi=\phi^{u} \in \Phi^{u}$;

(b) $I \neq \emptyset$ and $H \neq \emptyset$, so for some $i \in U$, there exists $x_{p}^{i} \in L_{p} \backslash\left\{0, l_{p}\right\}$ such that $\phi\left(1_{-i, p}, 0_{i}, x_{p}^{i}\right)=1$

(c) $I \neq \emptyset$ and $H=\emptyset$, so for each $i \in U$, there exists $x_{p}^{i} \in L_{p} \backslash\left\{0, l_{p}\right\}$ such that $\phi\left(1_{-i, p}, 0_{i}, x_{p}^{i}\right)=1$.

2. Suppose that for some $i \in U, \phi\left(1_{-i, p}, 0_{i}, l_{p}\right)=1$, so $J \neq \emptyset$. We have to consider three cases:

(d) $H=U \backslash J$, so for each $i \in U \backslash J$, for each $x_{p} \in L_{p} \backslash\{0\}, \phi\left(1_{-i}, 0_{i}, x_{p}\right)=0$;

(e) $I=U \backslash J$, so for some $i \in U \backslash J$, there exists $x_{p}^{i} \in L_{p} \backslash\left\{0, l_{p}\right\}$ such that $\phi\left(1_{-i, p}, 0_{i}, x_{p}^{i}\right)=1$

(f) $I \neq \emptyset$ and $H \neq \emptyset$, so for some $i \in U \backslash J$, there exists $x_{p}^{i} \in L_{p} \backslash\left\{0, l_{p}\right\}$ such that $\phi\left(1_{-i, p}, 0_{i}, x_{p}^{i}\right)=1$

To obtain a contradiction we have to show that $\phi$ is not core compatible for cases 1.(b)-2.(f). To this end, we have to consider each type of mapping of rights $\phi^{1 .(b)}-\phi^{2 \cdot(f)}$ corresponding to each case. For each type of mapping of rights we have to find an instance $P \in \mathcal{P}$ such that the core of the associated game is empty. We will consider the social cost problem $P=\left(N, L_{p}, B_{p},\left(D_{i}\right)_{i \in U}\right) \in \mathcal{P}$ defined as

$\forall k \in L_{p}=\{0,1,2,3\}, \quad B_{p}(k)=7 \sqrt{k}, \quad D_{1}(k)=5.5 k, \quad D_{2}(k)=2 k, \quad D_{i}(k)=0, \quad i \in U \backslash\{1,2\}$.

Also we will focus on participation profiles $x \in L$ where at least $n-1$ agents are active. Therefore we will consider the balanced collection $\mathcal{B}$ of elements of $L$ defined as:

$$
\mathcal{B}:=\{x \in L:|\mathcal{S}(x)|=n-1\}
$$

with the balancing weights defined as follows:

$$
\mu(x)=\frac{l_{p}}{n-1}, \text { if } \mathcal{S}(x)=U ; \text { and } \mu(x)=\frac{1}{n-1} \text { otherwise. }
$$

Consider any mapping of rights $\phi^{1 .(b)}$ such that $H \neq \emptyset$ and $I \neq \emptyset$. Assume that $1 \in H$, $2 \in I$ and for each $i \in I, \tilde{x}_{p}^{i}=2$. For participation profile $x \in L$ where $|\mathcal{S}(x)|=\{n-1, n\}$, we have: 
1. for each $x_{p} \in L_{p} \backslash\{0\}, v_{\phi^{1 .(b)}, P}\left(1_{-p}, x_{p}\right)=0$,

2. $v_{\phi^{1 .(b)}, P}\left(1_{-p}, 0_{p}\right)=0$,

3. $v_{\phi^{1 .(b)}, P}\left(1_{-2, p}, 0_{2}, 1_{p}\right)=1.5$

4. for any $x_{p} \in L_{p} \backslash\{0,1\}, v_{\phi^{1 .(b)}, P}\left(1_{-2, p}, 0_{2}, x_{p}\right)=0$,

5. for each $h \in H$, for each $x_{p} \in L_{p} \backslash\{0\}, v_{\phi^{1 .(b)}, P}\left(1_{-h, p}, 0_{h}, x_{p}\right)=0$,

6. for each $i \in I \backslash\{2\}$, for each $x_{p} \in L_{p} \backslash\{0\}, v_{\phi^{1 .(b)}, P}\left(1_{-i, p}, 0_{i}, x_{p}\right)=v_{\phi^{1 .(b)}, P}\left(1_{-p}, x_{p}\right)=0$.

Simple computations give:

$$
\sum_{x \in \mathcal{B}} \mu(x) v_{\phi^{1 .(b)}, P}(x)=\frac{1.5}{n-1}>0=\sum_{x_{p}=1}^{3} v\left(1_{-p}, x_{p}\right) .
$$

Therefore, by proposition 2.1 we have $\mathcal{C}\left(v_{\phi^{1 .(b)}, P}\right)=\emptyset$.

Consider any mapping of rights $\phi^{1 .(c)}$, then $I=U$. Furthermore assume that $\tilde{x}_{p}^{i}=2$ we have:

1. for each $x_{p} \in L_{p} \backslash\{0\}, v_{\phi^{1 .(c)}, P}\left(1_{-p}, x_{p}\right)=0$,

2. $v_{\phi^{1 .}(c), P}\left(1_{-p}, 0_{p}\right)=0$,

3. $v_{\phi^{1 .(c)}, P}\left(1_{-2, p}, 0_{2}, 1_{p}\right)=1.5$,

4. $v_{\phi^{1 .(c)}, P}\left(1_{-1, p}, 0_{1}, 1_{p}\right)=5$,

5. for each $i \in I \backslash\{1,2\}$, for each $x_{p} \in L_{p} \backslash\{0\}, v_{\phi^{1 .(c)}, P}\left(1_{-i, p}, 0_{i}, x_{p}\right)=v_{\phi^{1 .(c)}, P}\left(1_{-p}, x_{p}\right)=0$.

By taking the balanced collection $\mathcal{B}$ as defined above, simple computations give us:

$$
\sum_{x \in \mathcal{B}} \mu(x) v_{\phi^{1 .(c)}, P}(x)=\frac{6.5}{n-1}>0=\sum_{x_{p}=1}^{3} v\left(1_{-p}, x_{p}\right) .
$$

Again, by proposition 2.1 we have $\mathcal{C}\left(v_{\phi^{1 .(c)}, P}\right)=\emptyset$.

Consider any mapping of rights $\phi^{2 \cdot(d)}$ such that $J \neq \emptyset$ and $H=U \backslash J$. Assume that $1 \in H$ and $2 \in J$. Then we obtain,

1. for each $x_{p} \in L_{p} \backslash\{0\}, v_{\phi^{1 .(b)}, P}\left(1_{-p}, x_{p}\right)=0$,

2. $v_{\phi^{2 \cdot(d)}, P}\left(1_{-p}, 0_{p}\right)=0$,

3. for each $x_{p} \in L_{p} \backslash\{0\}, v_{\phi^{2 \cdot(d)}, P}\left(1_{-2, p}, 0_{2}, x_{p}\right)=1.5$,

4. for each $h \in H$, for each $x_{p} \in L_{p} \backslash\{0\}, v_{\phi^{2 .(d)}, P}\left(1_{-h, p}, 0_{h}, x_{p}\right)=0$,

5. for each $j \in J \backslash\{2\}$, for each $x_{p} \in L_{p} \backslash\{0\}, v_{\phi^{2 .(d)}, P}\left(1_{-j, p}, 0_{j}, x_{p}\right)=v_{\phi^{2 .(d)}, P}\left(1_{-p}, x_{p}\right)=0$. 
By taking the balanced collection $\mathcal{B}$ as defined above, simple computations give us:

$$
\sum_{x \in \mathcal{B}} \mu(x) v_{\phi^{2 \cdot(d)}, P}(x)=\frac{4.5}{n-1}>0=\sum_{x_{p}=1}^{3} v\left(1_{-p}, x_{p}\right) .
$$

Therefore, by proposition 2.1 we have $\mathcal{C}\left(v_{\phi^{2 .(d)}, P}\right)=\emptyset$.

Consider any mapping of rights $\phi^{2 .(e)}$ such that $J \neq \emptyset$ and $I=U \backslash J$. Assume that $2 \in I$ with $\tilde{x}_{p}^{i}=2$ and $1 \in J$. Then we obtain,

1. for each $x_{p} \in L_{p} \backslash\{0\}, v_{\phi^{1} \cdot(c), P}\left(1_{-p}, x_{p}\right)=0$,

2. $v_{\phi^{2 .(e)}, P}\left(1_{-p}, 0_{p}\right)=0$,

3. $v_{\phi^{2 .(e)}, P}\left(1_{-2, p}, 0_{2}, 1_{p}\right)=1.5$,

4. for each $x_{p} \in L_{p} \backslash\{0\}, v_{\phi^{2 .(e)}, P}\left(1_{-1, p}, 0_{1}, x_{p}\right)=5$,

5. for each $i \in I \backslash\{2\}$, for each $x_{p} \in L_{p} \backslash\{0\}, v_{\phi^{2 .(e)}, P}\left(1_{-i, p}, 0_{i}, x_{p}\right)=v_{\phi^{2}(e), P}\left(1_{-p}, x_{p}\right)=0$.

$$
\sum_{x \in \mathcal{B}} \mu(x) v_{\phi^{2 \cdot(e), P}}(x)=\frac{16.5}{n-1}>0=\sum_{x_{p}=1}^{3} v\left(1_{-p}, x_{p}\right) .
$$

Therefore, by proposition 2.1 we have $\mathcal{C}\left(v_{\phi^{2 \cdot(e), P}}\right)=\emptyset$.

Finally, consider any mapping of rights $\phi^{2 \cdot(f)}$ such that $J \neq \emptyset, I \neq \emptyset$ and $H \neq \emptyset$. Assume that $2 \in I$ and $1 \in H$. Then we obtain $v_{\phi^{2 .(f)}, P}=v_{\phi^{1 .(b)}, P}$, therefore by proposition 2.1 we have $\mathcal{C}\left(v_{\phi^{2 \cdot(f), P}}\right)=\emptyset$.

From 1(a)-2(f), we conclude that if $\phi \in \Phi \backslash \tilde{\Phi}$ is core compatible then $\phi \in \Phi^{u}$. Hence, only mappings of rights $\phi \in \tilde{\Phi}$ and $\phi \in \Phi^{u}$ satisfy core compatibility.

\section{References}

Aivazian VA, Callen JL (1981) The coase theorem and the empty core. The Journal of Law and Economics 24(1):175-181

Ambec S, Kervinio Y (2016) Cooperative decision-making for the provision of a locally undesirable facility. Social Choice and Welfare 46(1):119-155

Anderlini L, Felli L (2001) Costly bargaining and renegotiation. Econometrica 69(2):377-411

Bloch F, Van den Nouweland A (2014) Expectation formation rules and the core of partition function games. Games and Economic Behavior 88:339-353

Bondareva O (1962) The theory of the core in an n-person game. Vestnik Leningrad Univ $13: 141-142$

Chipman JS, Tian G (2012) Detrimental externalities, pollution rights, and the "coase theorem". Economic Theory 49(2):309-327 
Coase RH (1960) The problem of social cost. Journal of Law and Economics 3(2):1-44

Ellingsen T, Paltseva E (2016) Confining the coase theorem: contracting, ownership, and free-riding. The Review of Economic Studies 83(2):547-586

Funaki Y, Yamato T (1999) The core of an economy with a common pool resource: A partition function form approach. International Journal of Game Theory 28(2):157-171

Gonzalez S, Marciano A, Solal P (2019) The social cost problem, rights, and the (non) empty core. Journal of Public Economic Theory 21(2):347-365

Grabisch M, Xie L (2007) A new approach to the core and weber set of multichoice games. Mathematical Methods of Operations Research 66(3):491-512

Hsiao CR, Raghavan T (1992) Monotonicity and dummy free property for multi-choice cooperative games. International Journal of Game Theory 21(3):301-312

Hsiao CR, Raghavan T (1993) Shapley value for multichoice cooperative games, i. Games and economic behavior 5(2):240-256

Hurwicz L (1995) What is the coase theorem? Japan and the World Economy 7(1):49-74

Hwang YA, Liao YH (2011) The multi-core, balancedness and axiomatizations for multichoice games. International Journal of Game Theory 40(4):677-689

van den Nouweland A, Tijs S, Potters J, Zarzuelo J (1995) Cores and related solution concepts for multi-choice games. Zeitschrift für Operations Research 41(3):289-311

Pigou AC (1920) The Economics of Welfare. London: Macmillan.

Shapley LS (1967) On balanced sets and cores. Naval research logistics quarterly 14(4):453460

Shapley LS, Shubik M (1969) On the core of an economic system with externalities. The American Economic Review 59(4):678-684

Stigler GJ (1966) The theory of price. New York: Macmillan.

Zhao J (2018) A reexamination of the coase theorem. The Journal of Mechanism and Institution Design 3(1):111-132 